\title{
LARGE DEVIATIONS OF THE LYAPUNOV EXPONENT AND LOCALIZATION FOR THE 1D ANDERSON MODEL
}

\author{
SVETLANA JITOMIRSKAYA, XIAOWEN ZHU
}

\begin{abstract}
The proof of Anderson localization for the 1D Anderson model with arbitrary (e.g. Bernoulli) disorder, originally given by Carmona-KleinMartinelli in 1987, is based in part on the multi-scale analysis. Later, in the 90 s, it was realized that for one-dimensional models with positive Lyapunov exponents some parts of multi-scale analysis can be replaced by considerations involving subharmonicity and large deviation estimates for the corresponding cocycle, leading to nonperturbative proofs for 1D quasiperiodic models. In this paper we present a short proof along these lines, for the Anderson model. To prove dynamical localization we also develop a uniform version of Craig-Simon's bound that works in high generality and may be of independent interest.
\end{abstract}

\section{INTRODUCTION}

Anderson localization for the Anderson model can be proved in several different ways if the common distribution of the i.i.d.r.v's is absolutely continuous. Without that condition (or at least some Hölder regularity) it remains an open question for $d \geqslant 2$, and the number of approaches that work for $d=1$ also drops dramatically. Such is the situation, for example, for the Bernoulli-Anderson model. Anderson localization for arbitrary 1D disorder was first proved in [1]. The approach was based on certain regularity of the Lyapunov exponents coming from the (analysis around) the Furstenberg theorem to obtain an analogue of Wegner's lemma (automatic in the absolutely continuous case). After that the proof was reduced to multi-scale analysis, with initial scale coming again from the positive Lyapunov exponent. Another argument was later presented in [2, where an approach to positivity and regularity of the Lyapunov exponent using replica trick was given, again reducing the proof to multi-scale analysis. Multi-scale analysis is a method that allows to achieve Green's function decay and ultimately localization from high probability of decay at the initial scale. It works in a variety of settings. Originally developed by Frohlich and Spencer [3], it was significantly simplified in 4 . but remains somewhat involved. It should be noted that in the multidimensional case no shortcuts such as Furstenberg theorem or replica trick are available, and the multi-scale analysis is used to reach conclusions analogous to the positivity of the Lyapunov exponent simultaneously with the proof of localization. Yet in the one-dimensional case positivity of the Lyapunov exponent essentially provides the averaged decay statement, thus a large portion of the conclusion of the multi-scale analysis, making its machinery seem redundant.

A method to effectively exploit positive Lyapunov exponent for a localization proof based on the analysis of the large deviation set for the Lyapunov exponent was first developed in [5] for the almost Mathieu operator, initiating what was 
later called a non-perturbative approach, in contrast with earlier proofs based on some form of multi-scale analysis 6, 7,. A robust method based on subharmonic function theory and the theory of semianalytic sets was then developed in $[8$ and other papers summarized in 9], to conclude localization from positive Lyapunov exponents for analytic quasiperiodic and some other deterministic potentials. The fact that those ideas can be applicable also to the Anderson model was mentioned in some talks by one of the authors circa 2000, but the details were never developed. One goal of this paper is to obtain a proof of Anderson localization for the 1D Anderson model in the spirit of [5] but with appropriate simplifications due to randomness.

Another proof, also based on large deviations and also avoiding multi-scale analysis was recently developed in [10. The proof of [10] is based on deterministic ideas close to the ones in [11, which we believe may be somewhat more complicated than needed for the random case. We mention that yet another, purely dynamical, proof of localization for the 1D Anderson model appears in [12].

One ingredient in our simple argument for spectral localization, Theorem 3.3 is Craig-Simon's upper bound based on subharmonicity of the Lyapunov exponent 13, a statement that holds for any ergodic potential. In order to prove dynamical localization we need a uniform in energy and quantitative version of this statement, that we prove for general ergodic potentials satisfying certain large deviation bounds, a result that could be of independent interest. We note that our proof does not explicitly use subharmonicity.

The rest of this paper is organized as follows. Section 2 contains the preliminaries, the statement of the spectral localization result, Theorem 2.1, and its quick reduction to Theorem 2.2, We then prove the preparatory Lemmas [3.1, 3.2, 3.5] and Corollary 3.4 in Section 3 . Then we complete the proof of Theorem 2.2 in Section 4. Our proof effectively establishes a more precise result, Theorem 4.1, which in turn immediately implies the Lyapunov behavior at all eigenvalues, Theorem 4.2. We formulate and prove the general uniform Craig-Simon-type statement in Section [5 and use it in Section 6 to prove dynamical localization.

\section{Preliminaries}

The one dimensional Anderson model is given by a discrete Schrödinger operators $H_{\omega}$

$$
\left(H_{\omega} \Psi\right)(n)=\Psi(n+1)+\Psi(n-1)+\omega_{n} \Psi(n),
$$

where $\omega_{n} \in \mathbb{R}$ are independent identically distributed random variables with common Borel probability distribution $\mu$. We will assume that $S \subset \mathbb{R}$, the topological support of $\mu$, is compact, and contains at least two points. We will denote the probability space $\Omega=S^{\mathbb{Z}}$, with elements $\left\{\omega_{n}\right\}_{n \in \mathbb{Z}} \in \Omega$. Denote $\mu^{\mathbb{Z}}$ as $\mathbb{P}$. Let $\mathbb{P}_{[a, b]}$ be $\mu^{[a, b] \cap \mathbb{Z}}$ on $S^{[a, b] \cap \mathbb{Z}}$. Aldo let $T$ be the shift $T \omega_{i}=\omega_{i-1}$. Finally, we denote Lebesgue measure on $\mathbb{R}$ by $m$. We say that $H_{\omega}$ has spectral localization in $I$ if for a.e. $\omega, H_{\omega}$ has only pure point spectrum in $I$ and its eigenfunctions $\Psi(n)$ decay exponentially in $n$.

Definition 1. We call $E$ a generalized eigenvalue (g.e.), if there exists a nonzero polynomially bounded function $\Psi(n)$ such that $H_{\omega} \Psi=E \Psi$. We call $\Psi(n)$ a generalized eigenfunction. 
Since the set of g.e. supports the spectral measure of $H_{\omega}$ (e.g. [14), we only need to show:

Theorem 2.1. For a.e. $\omega$, for every g.e. E, the corresponding generalized eigenfunction $\Psi_{\omega, E}(n)$ decays exponentially in $n$.

For $[a, b]$ an interval, $a, b \in \mathbb{Z}$, define $H_{[a, b], \omega}$ to be operator $H_{\omega}$ resticted to $[a, b]$ with zero boundary conditions outside $[a, b]$. Note that it can be expressed as a $" b-a+1 "$-dimensional matrix. The Green's function for $H_{\omega}$ restricted to $[a, b]$ with energy $E \notin \sigma_{[a, b], \omega}$ is

$$
G_{[a, b], E, \omega}=\left(H_{[a, b], \omega}-E\right)^{-1}
$$

Note that this can also be expressed as a " $b-a+1$ "-dimensional matrix. Denote its $(x, y)$ entry as $G_{[a, b], E, \omega}(x, y)$.

It is well known that

$$
\Psi(x)=-G_{[a, b], E, \omega}(x, a) \Psi(a-1)-G_{[a, b], E, \omega}(x, b) \Psi(b+1), \quad x \in[a, b]
$$

and we have

$$
\sigma:=\sigma\left(H_{\omega}\right)=[-2,2]+S \quad \text { a.e.w. }
$$

Definition 2. For $c>0, n \in \mathbb{Z}$, we say $x \in \mathbb{Z}$ is $(c, n, E, \omega)$-regular, if

$$
\begin{aligned}
& G_{[x-n, x+n], E, \omega}(x, x-n) \leqslant e^{-c n} \\
& G_{[x-n, x+n], E, \omega}(x, x+n) \leqslant e^{-c n}
\end{aligned}
$$

Otherwise, we call it $(c, n, E, \omega)$-singular.

By (2.2) and definition 2, Theorem 2.1 follows from

Theorem 2.2. There exists $\Omega_{0}$ with $\mathbb{P}\left(\Omega_{0}\right)=1$, such that for every $\tilde{\omega} \in \Omega_{0}$, for any g.e. $\tilde{E}$ of $H_{\tilde{\omega}}$, there exist $N=N(\tilde{E}, \tilde{\omega}), C=C(\tilde{E})$, such that for every $n>N$, $2 n, 2 n+1$ are $(C, n, \tilde{E}, \tilde{\omega})$-regular.

Some other standard basic settings are below. Denote

$$
P_{[a, b], E, \omega}=\operatorname{det}\left(H_{[a, b], E, \omega}-E\right), a \leqslant b
$$

If $a>b$, let $P_{[a, b], E, \omega}=1$. Then

$$
\left|G_{[a, b], E, \omega}(x, y)\right|=\frac{\left|P_{[a, x-1], E, \omega} P_{[y+1, b], E, \omega}\right|}{\left|P_{[a, b], E, \omega}\right|}, \quad x \leqslant y
$$

If we denote the transfer matrix $T_{[a, b], E, \omega}$ as the matrix such that

$$
\left(\begin{array}{c}
\Psi(b) \\
\Psi(b-1)
\end{array}\right)=T_{[a, b], E, \omega}\left(\begin{array}{c}
\Psi(a) \\
\Psi(a-1)
\end{array}\right)
$$

where $\Psi$ solves $H_{\omega} \Psi=E \Psi$, then

$$
T_{[a, b], E, \omega}=\left(\begin{array}{cc}
P_{[a, b], E, \omega} & -P_{[a+1, b], E, \omega} \\
P_{[a, b-1], E, \omega} & -P_{[a+1, b-1], E, \omega}
\end{array}\right)
$$

The Lyapunov exponent exists by Kingman's subadditive ergodic theorem and is given by

$$
\gamma(E)=\lim _{n \rightarrow \infty} \frac{1}{n} \int_{0}^{1} \log \left\|T_{[0, n], E, \omega}\right\| d \mathbb{P}(\omega)=\lim _{n \rightarrow \infty} \frac{1}{n} \log \left\|T_{[0, n], E, \omega}\right\|, \quad \text { a.e.w. }
$$


Let $\nu=\inf _{E \in \sigma} \gamma(E)$. By the Furstenberg's theorem $\nu>0$. It follows from (2.4) that the desired exponential decay of the Green's function can be achieved if all the $P_{[a, b]}$ in (2.4) behave as $e^{(b-a) \gamma(E)}$, thus leading to the study of deviations of $\ln P_{[a, b]}$ from its mean. In fact, the key estimates underlying the analysis of [1] are precisely large deviation bounds for the Lyapunov exponent due to Le Page [15]. Here we will use a corresponding statement for the matrix elements [16]

Lemma 2.3 ("uniform-LDT"). For any $\epsilon>0$, there exists $\eta=\eta(\epsilon)>0$ such that, there exists $N_{0}=N_{0}(\epsilon)$, such that for every $b-a>N_{0}$, and any $E$ in a compact set,

$$
\mathbb{P}\left\{\omega:\left|\frac{1}{b-a+1} \log \left\|P_{[a, b], E, \omega}\right\|-\gamma(E)\right| \geqslant \epsilon\right\} \leqslant e^{-\eta(b-a+1)}
$$

It will also be convenient to use the general subharmonicity upper bound due to Craig-Simon 13 .

Theorem 2.4 (Craig-Simon [13]). For a.e. $\omega$ for all E, we have

$$
\varlimsup_{n \rightarrow \infty} \frac{\log \left\|T_{[0, n], E, \omega}\right\|}{n+1} \leqslant \gamma(E)
$$

\section{MAIN LEMMAS}

Denote

$$
\begin{aligned}
& B_{[a, b], \epsilon}^{+}=\left\{(E, \omega):\left|P_{[a, b], E, \omega}\right| \geqslant e^{(\gamma(E)+\epsilon)(b-a+1)}\right\} \\
& B_{[a, b], \epsilon}^{-}=\left\{(E, \omega):\left|P_{[a, b], E, \omega}\right| \leqslant e^{(\gamma(E)-\epsilon)(b-a+1)}\right\}
\end{aligned}
$$

and denote $B_{[a, b], \epsilon, E}^{ \pm}=\left\{\omega:(E, \omega) \in B_{[a, b], \epsilon}^{ \pm}\right\}, B_{[a, b], \epsilon, \omega}^{ \pm}=\left\{E:(E, \omega) \in B_{[a, b], \epsilon}^{ \pm}\right\}$, $B_{[a, b], *}=B_{[a, b], *}^{+} \cup B_{[a, b], *}^{-}$.

Let $E_{j,\left(\omega_{a}, \cdots, \omega_{b}\right)}$ be eigenvalues of $H_{[a, b], \omega}$ with $\left.\omega\right|_{[a, b]}=\left(\omega_{a}, \cdots, \omega_{b}\right)$.

Large deviation theorem gives us the estimate that for all $E, a, b, \epsilon$

$$
\mathbb{P}\left(B_{[a, b], \epsilon, E}^{ \pm}\right) \leqslant e^{-\eta(b-a+1)}
$$

Assume $\epsilon=\epsilon_{0}<\frac{1}{8} \nu$ is fixed for now, so we omit it from the notations until Lemma 3.5. Let $\eta_{0}=\eta\left(\epsilon_{0}\right)$ be the corresponding parameter from Lemma 2.3

Lemma 3.1. For $n \geqslant 2$, if $x$ is $\left(\gamma(E)-8 \epsilon_{0}, n, E, \omega\right)$-singular, then

$$
(E, \omega) \in B_{[x-n, x+n]}^{-} \cup B_{[x-n, x]}^{+} \cup B_{[x, x+n]}^{+}
$$

Remark 1. Note that from (3.3), for all $E, x, n \geqslant 2$,

$$
\mathbb{P}\left(B_{[x-n, x+n], E}^{-} \cup B_{[x-n, x], E}^{+} \cup B_{[x, x+n], E}^{+}\right) \leqslant 3 e^{-\eta_{0}(n+1)}
$$

Proof. Follows imediately from the definition of singularity and (2.4). 2.2

Now we will use the following three lemmas to find the proper $\Omega_{0}$ for Theorem

Lemma 3.2. Let $0<\delta_{0}<\eta_{0}$. For a.e. $\omega$ (we denote this set as $\Omega_{1}$ ), there exists $N_{1}=N_{1}(\omega)$, such that for every $n>N_{1}$,

$$
\max \left\{m\left(B_{[n+1,3 n+1], \omega}^{-}\right), m\left(B_{[-n, n], \omega}^{-}\right)\right\} \leqslant e^{-\left(\eta_{0}-\delta_{0}\right)(2 n+1)}
$$


Proof. By (3.3),

$$
\begin{gathered}
m \times \mathbb{P}\left(B_{[n+1,3 n+1]}^{-}\right) \leqslant m(\sigma) e^{-\eta_{0}(2 n+1)} \\
m \times \mathbb{P}\left(B_{[-n, n]}^{-}\right) \leqslant m(\sigma) e^{-\eta_{0}(2 n+1)}
\end{gathered}
$$

If we denote

$$
\begin{gathered}
\Omega_{\delta_{0}, n,+}=\left\{\omega: m\left(B_{[n+1,3 n+1], \omega}^{-}\right) \leqslant e^{-\left(\eta_{0}-\delta_{0}\right)(2 n+1)}\right\} \\
\Omega_{\delta_{0}, n,-}=\left\{\omega: m\left(B_{[-n, n], \omega}^{-}\right) \leqslant e^{-\left(\eta_{0}-\delta_{0}\right)(2 n+1)}\right\}
\end{gathered}
$$

We have by Tchebyshev,

$$
\mathbb{P}\left(\Omega_{\delta_{0}, n, \pm}^{c}\right) \leqslant m(\sigma) e^{-\delta_{0}(2 n+1)} .
$$

By Borel-Cantelli lemma, we get for a.e. $\omega$,

$$
\max \left\{m\left(B_{[n+1,3 n+1], \omega}^{-}\right), m\left(B_{[-n, n], \omega}^{-}\right)\right\} \leqslant e^{-\left(\eta_{0}-\delta_{0}\right)(2 n+1)},
$$

for $n>N_{1}(\omega)$.

Remark 2. Note that we can actually shift the operator and use center point $l$ instead of 0 . Then we will get $\Omega_{1}(l)$ instead of $\Omega_{1}, N_{1}(l, \omega)$ instead of $N_{1}(\omega)$. And if we pick $N_{1}(l, \omega)$ in the theorem as the smallest integer satisfying the conclusion, we can estimate when we will have $N_{1}(l, \omega) \leqslant \ln ^{2}|l|$, which is very useful in the proof for dynamical localization in section 6 .

The next results follows from :

Theorem 3.3. For a.e. $\omega$ (we denote this set as $\Omega_{2}$ ), for all $E$, we have

$$
\begin{gathered}
\max \left\{\varlimsup_{n \rightarrow \infty} \frac{\log \left\|T_{[-n, 0], E, \omega}\right\|}{n+1}, \varlimsup_{n \rightarrow \infty} \frac{\log \left\|T_{[0, n], E, \omega}\right\|}{n+1}\right\} \leqslant \gamma(E) \\
\max \left\{\varlimsup_{n \rightarrow \infty} \frac{\log \left\|T_{[n+1,2 n+1], E, \omega}\right\|}{n+1}, \varlimsup_{n \rightarrow \infty} \frac{\log \left\|T_{[2 n+1,3 n+1], E, \omega}\right\|}{n+1}\right\} \leqslant \gamma(E)
\end{gathered}
$$

Remark 3. (3.5) is a direct reformulation of the result of [13], Theorem 2.4, while (3.6) follows by exactly the same proof.

Corollary 3.4. For every $\omega \in \Omega_{2}$, for every $E$, there exists $N_{2}=N_{2}(\omega, E)$, such that for every $n>N_{2}$,

$$
\begin{aligned}
& \max \left\{\left\|T_{[-n, 0], E, \omega}\right\|,\left\|T_{[0, n], E, \omega}\right\|\right\}<e^{(\gamma(E)+\epsilon)(n+1)} \\
& \max \left\{\left\|T_{[n+1,2 n+1], E, \omega}\right\|,\left\|T_{[2 n+1,3 n+1], E, \omega}\right\|\right\}<e^{(\gamma(E)+\epsilon)(n+1)}
\end{aligned}
$$

Lemma 3.5. Let $\epsilon>0, K>1$, For a.e. $\omega$ (we denote this set as $\Omega_{3}=\Omega_{3}(\epsilon, K)$ ), there exists $N_{3}=N_{3}(\omega)$, so that for every $n>N_{3}$, for every $E_{j,\left(\omega_{n+1}, \cdots, \omega_{3 n+1}\right)}$, for every $y_{1}, y_{2}$ satisfying $-n \leqslant y_{1} \leqslant y_{2} \leqslant n,\left|-n-y_{1}\right| \geqslant \frac{n}{K}$, and $\left|n-y_{2}\right| \geqslant \frac{n}{K}$, we have $E_{j,\left(\omega_{n+1}, \cdots, \omega_{3 n+1}\right)} \notin B_{\left[-n, y_{1}\right], \epsilon, \omega} \cup B_{\left[y_{2}, n\right], \epsilon, \omega}$.

Remark 4. Note that $\epsilon$ and $K$ are not fixed yet, we're going to determine them later in section 4 
Proof. Let $\overline{\mathbb{P}}$ be the probability that there are some $y_{1}, y_{2}, j$ with

$$
E_{j,\left(\omega_{n+1}, \cdots, \omega_{3 n+1}\right)} \in B_{\left[-n, y_{1}\right], \epsilon, \omega} \cup B_{\left[y_{2}, n\right], \epsilon, \omega} .
$$

Note that for any fixed $\omega_{c}, \cdots, \omega_{d}$, with $[c, d] \cap[a, b]=\varnothing$, by independence,

$$
\mathbb{P}\left(B_{\left.[a, b], \epsilon, E_{j,\left(\omega_{c}, \cdots, \omega_{d}\right)}\right)}\right)=\mathbb{P}_{[a, b]}\left(B_{[a, b], \epsilon, E_{j,\left(\omega_{c}, \cdots, \omega_{d}\right)}}\right) \leqslant e^{-\eta_{0}(b-a+1)}
$$

Applying to $[a, b]=\left[-n, y_{1}\right]$ or $\left[y_{2}, n\right],[c, d]=[n+1,3 n+1]$ and integrating over $\omega_{-n}, \cdots, \omega_{y_{1}}$ or $\omega_{y_{2}}, \cdots, \omega_{n}$, we get

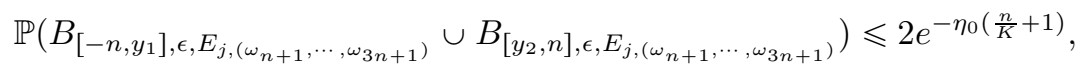

so

$$
\overline{\mathbb{P}} \leqslant(2 n+1)^{3} 2 e^{-\eta_{0}\left(\frac{n}{K}+1\right)}
$$

Thus by Borel-Cantelli, we get the result.

Remark 5. Similar to remark 2, we can get $\Omega_{3}(l), N_{3}(l, \omega)$ for an operator shifted by $\ell$ instead, and get the result that for a.e. $\omega$ (we denote this set as $\Omega_{N_{3}}$ ), there exists $L_{3}(\omega)$, such that for any $|l|>L_{3}, N_{3}(l, \omega) \leqslant \ln ^{2}|l|$. This will be of use in section 6 for proving dynamical localization.

\section{Proof of Theorem 2.2}

We will only provide a proof that $2 n+1$ is $(c, n, E, \omega)$-regular, the argument for $2 n$ being similar.

Proof. Let $\epsilon$ be small enough such that

$$
\epsilon<\min \left\{\left(\eta_{0}-\delta_{0}\right) / 3, \nu\right\} .
$$

Now let

$$
L:=e^{\left(\eta_{0}-\delta_{0}-\epsilon\right)}>1,
$$

and note that since $S$ is bounded, by (2.3) we have there exists $M>0$, such that

$$
\left|P_{[a, b], E, \omega}\right|<M^{(b-a+1)}, \quad \forall E \in \sigma, \omega
$$

Pick $K$ big enough such that

$$
M^{\frac{1}{K}}<L
$$

Let $\sigma>0$ be such that

$$
M^{\frac{1}{K}} \leqslant L-\sigma<L
$$

Let $\Omega_{0}=\Omega_{1} \cap \Omega_{2} \cap \Omega_{3}(\epsilon, K)$. Pick $\tilde{\omega} \in \Omega_{0}$, and take $\tilde{E}$ a g.e. for $H_{\tilde{\omega}}$, with $\Psi$ the corresponding generalized eigenfunction. Without loss of generality assume $\Psi(0) \neq$ 0 . Then there exists $N_{4}$, such that for every $n>N_{4}, 0$ is $\left(\gamma(\tilde{E})-8 \epsilon_{0}, n, \tilde{E}, \tilde{\omega}\right)$ singular.

For $n>N_{0}=\max \left\{N_{1}(\tilde{\omega}), N_{2}(\tilde{\omega}, \tilde{E}), N_{3}(\tilde{\omega}), N_{4}(\tilde{\omega}, \tilde{E})\right\}$, assume $2 n+1$ is $(\gamma(\tilde{E})-$ $\left.8 \epsilon_{0}, n, \tilde{E}, \tilde{\omega}\right)$-singular. Then both 0 and $2 n+1$ is $\left(\gamma(\tilde{E})-8 \epsilon_{0}, n, \tilde{E}, \tilde{\omega}\right)$-singular. So by Lemma 3.1 $\tilde{E} \in B_{[n+1,3 n+1], \epsilon_{0}, \tilde{\omega}}^{-} \cup B_{[n+1,2 n+1], \epsilon_{0}, \tilde{\omega}}^{+} \cup B_{[2 n+1,3 n+1], \epsilon_{0}, \tilde{\omega}}^{+}$. By Corollary 3.4 and (3.1), $\tilde{E} \notin B_{[n+1,2 n+1], \epsilon_{0}, \tilde{\omega}}^{+} \cup B_{[2 n+1,3 n+1], \epsilon_{0}, \tilde{\omega}}^{+}$, so it can only lie in $B_{[n+1,3 n+1], \epsilon_{0}, \tilde{\omega}}^{-}$.

Note that in (3.2), $P_{[n+1,3 n+1], E, \tilde{\omega}}$ is a polynomial in $E$ that has $2 n+1$ real zeros (eigenvalues of $H_{[n+1,3 n+1], \tilde{\omega}}$ ), which are all in $B=B_{[n+1,3 n+1], \epsilon, \tilde{\omega}}^{-}$. Thus $B$ 
consists of less than or equal to $2 n+1$ intervals around the eigenvalues. $\tilde{E}$ should lie in one of them. By Lemma $3.2, m(B) \leqslant C e^{-\left(\eta_{0}-\delta_{0}\right)(2 n+1)}$. So there is some e.v. $E_{j,[n+1,3 n+1], \tilde{\omega}}$ of $H_{[n+1,3 n+1], \omega}$ such that

$$
\left|\tilde{E}-E_{j,[n+1,3 n+1], \tilde{\omega}}\right| \leqslant e^{-\left(\eta_{0}-\delta_{0}\right)(2 n+1)}
$$

By the same argument, there exists $E_{i,[-n, n], \tilde{\omega}}$, such that

$$
\left|\tilde{E}-E_{i,[-n, n], \tilde{\omega}}\right| \leqslant e^{-\left(\eta_{0}-\delta_{0}\right)(2 n+1)}
$$

Thus $\left|E_{i,[-n, n], \tilde{\omega}}-E_{j,[n+1,3 n+1], \tilde{\omega}}\right| \leqslant 2 e^{-\left(\eta_{0}-\delta_{0}\right)(2 n+1)}$. However, by Theorem 3.5 , one has $E_{j,[n+1,3 n+1], \tilde{\omega}} \notin B_{[-n, n], \epsilon, \tilde{\omega}}$, while $E_{i,[-n, n], \tilde{\omega}} \in B_{[-n, n], \epsilon, \tilde{\omega}}$ This will give us a contradiction below.

Since $\left|E_{i,[-n, n], \tilde{\omega}}-E_{j,[n+1,3 n+1], \tilde{\omega}}\right| \leqslant 2 e^{-\left(\eta_{0}-\delta_{0}\right)(2 n+1)}$ and $E_{i,[-n, n], \tilde{\omega}}$ is the e.v. of $H_{[-n, n], \tilde{\omega}}$

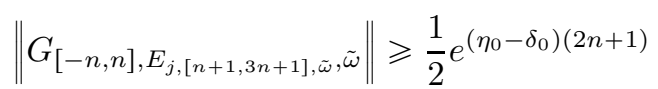

Thus there exist $y_{1}, y_{2} \in[-n, n]$ and such that

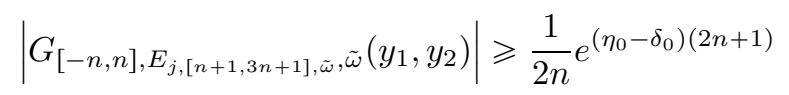

Let $E_{j}=E_{j,[n+1,3 n+1], \tilde{\omega}}$. We have $E_{j} \notin B_{[-n, n], \epsilon, \tilde{\omega}}$, thus

$$
\left|P_{[-n, n], \epsilon, E_{j}, \tilde{\omega}}\right| \geqslant e^{\left(\gamma\left(E_{j}\right)-\epsilon\right)(2 n+1)}
$$

so by (2.4),

$$
\left\|P_{\left[-n, y_{1}\right], \epsilon, E_{j} \tilde{\omega}} P_{\left[y_{2}, n\right], \epsilon, E_{j}, \tilde{\omega}}\right\| \geqslant \frac{1}{2 n} e^{\left(\eta_{0}-\delta_{0}\right)(2 n+1)} e^{\left(\gamma\left(E_{j}\right)-\epsilon\right)(2 n+1)}
$$

Then for the left hand side of (4.3), there are three cases:

(1) both $\left|-n-y_{1}\right|>\frac{n}{K}$ and $\left|n-y_{2}\right|>\frac{n}{K}$

(2) one of them is large, say $\left|-n-y_{1}\right|>\frac{n}{K}$ while $\left|n-y_{2}\right| \leqslant \frac{n}{K}$

(3) both small.

For (1),

$$
\frac{1}{2 n} e^{\left(\eta_{0}-\delta_{0}+\gamma\left(E_{j}\right)-\epsilon\right)(2 n+1)} \leqslant e^{2 n\left(\gamma\left(E_{j}\right)+\epsilon\right)}
$$

Since by our choice (4.1), $\eta_{0}-\delta_{0}+\gamma\left(E_{j}\right)-\epsilon>\gamma\left(E_{j}\right)+\epsilon$, for $n$ large enough, we get a contradiction.

For (2),

$$
\frac{1}{2 n} e^{\left(\eta_{0}-\delta_{0}+\gamma\left(E_{j}\right)-\epsilon\right)(2 n+1)} \leqslant e^{\left(\gamma\left(E_{j}\right)+\epsilon\right)(2 n+1)}(M)^{\frac{n}{K}}
$$

is in contradiction with (4.1) and (4.2)

For (3), with (4.1) and (4.2)

$$
\frac{1}{2 n} e^{\left(\eta_{0}-\delta_{0}+\gamma\left(E_{j}\right)-\epsilon\right)(2 n+1)} \leqslant M^{\frac{2 n}{K}} \leqslant(L-\sigma)^{2 n} \leqslant\left(e^{\left(\eta_{0}-\delta_{0}+\gamma\left(E_{j}\right)-\epsilon\right)}-\sigma\right)^{2 n},
$$

also a contradiction.

Thus our assumption that $2 n+1$ is not $\left(\gamma(\tilde{E})-8 \epsilon_{0}, n, \tilde{E}, \tilde{\omega}\right)$-regular is false. Theorem 2.2 follows.

Note that we have established the following more precise version of Theorem 2.2

Theorem 4.1. There exists $\Omega_{0}$ with $\mathbb{P}\left(\Omega_{0}\right)=1$, such that for every $\tilde{\omega} \in \Omega_{0}$, for any g.e. $\tilde{E}$ of $H_{\tilde{\omega}}$, and $\epsilon>0$, there exists $N=N(\tilde{E}, \tilde{\omega}, \epsilon)$, such that for every $n>N, 2 n, 2 n+1$ are $(\gamma(E)-\epsilon, n, \tilde{E}, \tilde{\omega})$-regular. 
It is a standard patching argument (e.g. proof of Theorem 3 in 5]) that this implies $\left|\Psi_{E}(n)\right| \leqslant C_{E, \epsilon} e^{-(\gamma(E)-\epsilon) n}$ for any $\epsilon>0$. Combined with Theorem 2.4 this immediately implies that we have Lyapunov behavior at every generalized eigenvalue.

Theorem 4.2. For a.e. $\omega$ for all generalized eigenvalues $E$, we have

$$
\lim _{n \rightarrow \infty} \frac{\log \left\|T_{[0, n], E, \omega}\right\|}{n+1}=\gamma(E)
$$

\section{Uniform and Quantitative Craig-Simon}

Craig-Simon theorem 2.4 implies that for a.e. $\omega$ and every $E \in \sigma$ there exists $N(\omega, E)$ such that for $n>N,\left\|T_{[0, n], E, \omega}\right\| \leqslant e^{(n+1)(\gamma(E)+\epsilon)}$. For the proof of dynamical localization one however needs a statement of this type with $N$ uniform in $E$. Such a statement is the goal of this section. We will show that it holds for any ergodic dynamical system satisfying the uniform LDT (Large Deviation Type) condition: Lemma 2.3. Thus this result has more general nature than the rest of the paper and may be of independent interest. In particular, it is applicable to quasiperiodic dynamics with Diophantine frequencies and analytic sampling functions. We note that uniform LDT condition can also be replaced by a combination of a pointwise LDT condition and continuity of the Lyapunov exponent.

We have:

Theorem 5.1. Let the ergodic family $H_{\omega}$ satisfy Lemma 2.3. Fix $\epsilon_{0}>0$. For a.e. $\omega$ (we denote this set as $\Omega_{2}=\Omega_{2}\left(\epsilon_{0}\right)$ ), there exists $N_{2}(\omega)$, such that for any $n>N_{2}(\omega), E \in \sigma$,

$$
\left|P_{[0, n], E, \omega}\right| \leqslant e^{\left(\gamma(E)+\epsilon_{0}\right)(n+1)}
$$

An immediate corollary is

Corollary 5.2. Let $H_{\omega}, \epsilon_{0}$ be as above. Then there exists $\Omega_{2}$ with $\mathbb{P}\left(\Omega_{2}\right)=1$, such that for $\omega \in \Omega_{2}$, there exists $N_{2}(\omega)$ such that

$\max \left\{\left|P_{[0, n], E, \omega}\right|,\left|P_{[-n, 0], E, \omega}\right|,\left|P_{[n+1,2 n+1], E, \omega}\right|,\left|P_{[2 n+1,3 n+1], E, \omega}\right|\right\} \leqslant e^{\left(\gamma(E)+3 \epsilon_{0}\right)(n+1)}$.

Thus we can replace Corollary 3.4 with this uniform version.

Proof. We start with the following

Lemma 5.3. Let $Q(x)$ be a polynomial of degree $n-1$. Let $x_{i}=\cos \frac{2 \pi(i+\theta)}{n}$, $0<\theta<1 / 2, i=1,2, \cdots, n$. If $Q\left(x_{i}\right) \leqslant a^{n}$, for all $i$, then $Q(x) \leqslant C n a^{n}$, for all $x \in[-1,1]$, where $C=C(\theta)$ is a constant.

Proof. By Lagrange interpolation, we have

$$
Q(x)=\sum_{i=1}^{n} Q\left(x_{i}\right) \prod_{j \neq i} \frac{x-x_{j}}{x_{i}-x_{j}}
$$

Note that

$\sum_{j \neq i} \ln \left|x_{i}-x_{j}\right|=\sum_{j \neq i}\left\{\ln \left|\sin \frac{\pi(i+j+2 \theta)}{n}\right|+\ln \left|\sin \frac{\pi(i-j)}{n}\right|+\ln 2\right\}=: A+B+(n-1) \ln 2$.

We will use the following lemma without giving a proof. 
Lemma 5.4 (Lemma 9.6 in [17]). Let $p$ and $q$ be relatively prime. Let $1 \leqslant k_{0} \leqslant q$ be such that

$$
\left|\sin 2 \pi\left(x+k_{0} p /(2 q)\right)\right|=\min _{1 \leqslant k \leqslant q}|\sin 2 \pi(x+k p /(2 q))|
$$

Then

$$
\ln q+\ln (2 / \pi)<\sum_{\substack{k=1 \\ k \neq k_{0}}}^{q} \ln |\sin 2 \pi(x+k p /(2 q))|+(q-1) \ln 2 \leqslant \ln q .
$$

For $B$, we take $p=1, q=n, x=-i /(2 n), k=j$. Then $k_{0}=i$, and we get

$$
B \geqslant \ln n+\ln (2 / \pi)-(n-1) \ln 2 .
$$

For $A$, we estimate by Lemma 5.4 with $p=1, q=n, x=(i+2 \theta) / 2 n, k=j$. If $k_{0}=j_{0}$ is the minimum term of $\ln \left|\sin \frac{\pi(i+j+2 \theta)}{n}\right|$, then

$$
A \geqslant \ln n+\ln (2 / \pi)-(n-1) \ln 2-\ln \left|\sin \frac{\pi(2 i+2 \theta)}{n}\right|+\ln \left|\sin \frac{\pi\left(i+j_{0}+2 \theta\right)}{n}\right|
$$

For $0<\theta<1 / 4$, we have

$$
\frac{\left|\sin \frac{\pi(2 i+2 \theta)}{n}\right|}{\left|\sin \frac{\pi\left(i+j_{0}+2 \theta\right)}{n}\right|}=\frac{\left|\sin \frac{\pi(2 i+2 \theta)}{n}\right|}{\left|\sin \frac{\pi \cdot 2 \theta}{n}\right|} \leqslant \frac{1}{\left|\sin \frac{\pi \cdot 2 \theta}{n}\right|}=O(n)
$$

Thus

$$
\sum_{j \neq i} \ln \left|x_{i}-x_{j}\right| \geqslant-(n-1) \ln 2+\ln n+C
$$

Writing $x=\cos \frac{2 \pi a}{n}$, by Lemma 5.4, we get

$$
\sum_{j \neq i} \ln \left|x-x_{j}\right| \leqslant-(n-1) \ln 2+2 \ln n+C
$$

Thus

and we have

$$
\prod_{j \neq i} \frac{x-x_{j}}{x_{i}-x_{j}} \leqslant C n
$$

$$
Q(x) \leqslant C n a^{n}
$$

Now we can finish the proof of Theorem 5.1

We know that $\sigma$ is compact, so contained in some bounded closed interval. Assume we are dealing with $[a, a+A]$. Unifrom LDT implies that $\gamma$ is a continuous function of $E$ [18]. Since $\gamma(E)$ is uniformly continuous, for any $\epsilon_{0}$, there exists $\delta_{0}$ such that

$$
\left|\gamma\left(E_{x}\right)-\gamma\left(E_{y}\right)\right| \leqslant \epsilon_{0}, \quad \text { if }\left|E_{x}-E_{y}\right| \leqslant \delta_{0} .
$$

Divide the interval $[a, a+A]$ into length- $\delta_{0}$ sub-intervals. There are $K=\left[A / \delta_{0}\right]+$ 1 of them (the last one may be shorter). Denote them as $I_{k}$, for $k=1, \cdots, K$. For $I_{k}=\left[E_{k, n}, E_{k+1, n}\right]$, let $E_{k 1, n}, \cdots, E_{k n, n}$ be distributed as in Lemma 5.3. Namely, set $E_{k i, n}=E_{k, n}+\left(x_{i}+1\right) \delta_{0} / 2$, where $x_{i}$ are as in Lemma 5.3, $0<\theta<1 / 2$. Note that for any $E_{x}, E_{y} \in\left[E_{k 1, n}, E_{k n, n}\right],\left|\gamma\left(E_{x}\right)-\gamma\left(E_{y}\right)\right| \leqslant \epsilon_{0}$. Since by the uniform-LDT condition

$$
\mathbb{P}\left(\left\{\omega: \exists i=1, \cdots, \text { n, s.t. }\left|P_{[0, n], E_{k i, n}, \omega}\right| \geqslant e^{\left(\gamma\left(E_{k i, n}\right)+\epsilon_{0}\right)(n+1)}\right\}\right) \leqslant n e^{-\eta_{0}(n+1)},
$$


by Borel-Cantelli, for a.e. $\omega$, (we denote this set as $\Omega(k)$ ), there exists $N(k, \omega)$, such that for all $n>N(k, \omega)$,

$$
\left|P_{[0, n], E_{k i, n}, \omega}\right| \leqslant e^{\left(\gamma\left(E_{k i, n}\right)+\epsilon_{0}\right)(n+1)}, \quad \forall i=1, \cdots, n .
$$

If we denote $\gamma_{k, n}=\inf _{E \in\left[E_{k 1, n}, E_{k n, n}\right]} \gamma(E)$, then by (5.3)

$$
\left|P_{[0, n], E_{k i, n}, \omega}\right| \leqslant e^{\left(\gamma\left(E_{k i, n}\right)+\epsilon_{0}\right)(n+1)} \leqslant e^{\left(\gamma_{k, n}+2 \epsilon_{0}\right)(n+1)}, \quad \forall i=1, \cdots, n .
$$

Let $M$ be big enough such that, for any $n>M, n^{c} \leqslant e^{\epsilon_{0}(n+1)}$. Thus by Lemma [5.3, applied to $Q(x)=P\left(E_{k, n}+\frac{(x+1) \delta_{0}}{2}\right)$, for $E \in\left[E_{k, n}, E_{k+1, n}\right], n>$ $\max \{N(k, \omega), M\}$,

$$
\left|P_{[0, n], E, \omega}\right| \leqslant n^{c} e^{\left(\gamma_{k, n}+2 \epsilon_{0}\right)(n+1)} \leqslant n^{c} e^{\left(\gamma(E)+2 \epsilon_{0}\right)(n+1)} \leqslant e^{\left(\gamma(E)+3 \epsilon_{0}\right)(n+1)}
$$

Let $\Omega_{2}=\bigcap_{k} \Omega(k), \tilde{N}(\omega)=\max _{k}\{N(k, \omega), M\}$. Then for any $n>\tilde{N}(\omega)$,

$$
\left|P_{[0, n], E, \omega}\right| \leqslant e^{\left(\gamma(E)+3 \epsilon_{0}\right)(n+1)}, \quad \forall E \in[a, a+A]
$$

This allows us to also obtain a quantitative version of Theorem [5.1 Assume the $N_{2}(\omega)$ in Theorem 5.1 is chosen to be the smallest satisfying the condition. Let $l \in \mathbb{Z}, N_{2}(l, \omega)=N_{2}\left(T^{l} \omega\right)$. Let $\bar{\Omega}_{2}=\bigcap_{l \in \mathbb{Z}} T^{l} \Omega_{2}$.

Lemma 5.5. For a.e. $\omega$ (we denote this set as $\tilde{\Omega}_{2}$ ), there exists $L_{2}=L_{2}(\omega)$, such that for all $|l|>L_{2}, N_{2}(l, \omega) \leqslant \ln ^{2}|l|$. In particular, if $n>\ln ^{2}|l|$, then

$$
\left|P_{[l, l+n], E, \omega}\right| \leqslant e^{\left(\gamma(E)+\epsilon_{0}\right)(n+1)}, \text { for all } E \in \sigma
$$

Proof. Let $\omega \in \bar{\Omega}_{2}, l \in \mathbb{Z}, k \in \mathbb{N}$. By Theorem 5.1, $\bar{\Omega}$ has full measure. We have

$$
\begin{aligned}
\mathbb{P}\left\{\omega: N_{2}(l, \omega) \geqslant k\right\} & \leqslant \sum_{n=k}^{\infty} \mathbb{P}\left\{\omega: N_{2}(l, \omega)=n\right\} \leqslant \sum_{n=k}^{\infty} \mathbb{P}\left(B_{[l, l+n-1], E}^{+}\right) \\
& \leqslant \sum_{n=k}^{\infty} C e^{-\left(\gamma(E)+\epsilon_{0}\right) n} \leqslant C e^{-\left(\gamma(E)+\epsilon_{0}\right) k}
\end{aligned}
$$

Thus

$$
\mathbb{P}\left\{\omega: N_{2}(l, \omega) \geqslant \ln ^{2}|l|\right\} \leqslant C e^{-\left(\gamma(E)+\epsilon_{0}\right)\left(\ln ^{2}|l|\right)}
$$

By Borel-Cantelli lemma, we get the result and the corresponding $\tilde{\Omega}_{2}$.

\section{Dynamical Localization}

Now we have established the spectral localization for 1-d Anderson model. With some more effort, we can get the dynamical localization. We say that $H_{\omega}$ exhibits dynamical localization if for a.e. $\omega$, for any $\epsilon>0$, there exists $\alpha=\alpha(\omega)>0$, $C=C(\epsilon, \omega)$, such that for all $x, y \in \mathbb{Z}$ :

$$
\sup _{t}\left|\left\langle\delta_{x}, e^{-i t H_{\omega}} \delta_{y}\right\rangle\right| \leqslant C_{\epsilon} e^{\epsilon|y|} e^{-\alpha|x-y|}
$$

According to [19], we only need to prove that for a.e. $\omega, H_{\omega}$ has SULE (SemiUniformly Localized Eigenfunction). We say $H$ has SULE if $H$ has a complete set $\left\{\varphi_{E}\right\}$ of orthonormal eigenfunctions, such that there is $\alpha>0$, and for each $\epsilon>0$, a $C_{\epsilon}$ such that for any eigenvalue $E$, there exists $l=l_{E} \in \mathbb{Z}$, such that

$$
\left|\varphi_{E}(x)\right| \leqslant C_{\epsilon} e^{\epsilon\left|l_{E}\right|} e^{-\alpha\left|x-l_{E}\right|}, \quad x \in \mathbb{Z}
$$


In fact, we will prove that $\left|\varphi_{E}(x)\right| \leqslant C_{\epsilon} e^{C \ln ^{2}\left(1+\left|l_{E}\right|\right)} e^{-\alpha\left|x-l_{E}\right|}$, see (6.3), (6.5). In order to do this, we need to modify Lemma 3.2. Lemma 3.5 using the same method as in Lemma 5.5. Assume the $N_{i}(\omega), i=1,3$ in Lemmas 3.2 3.5 are chosen to be the smallest parameters satisfying the condition. Let $l \in \mathbb{Z}, N_{i}(l, \omega)=N_{i}\left(T^{l} \omega\right)$. Let $\bar{\Omega}_{i}=\bigcap_{l \in \mathbb{Z}} T^{l} \Omega_{i}, i=1,3$.

Lemma 6.1. For a.e. $\omega$ (we denote this set as $\tilde{\Omega}_{1,3}$ ), there are $L_{1}(\omega), L_{3}(\omega)$ such that for any $|l|>\max \left\{L_{1}, L_{3}\right\}$,

$$
\max \left\{N_{1}(l, \omega), N_{3}(l, \omega)\right\} \leqslant \ln ^{2}|l|
$$

Proof. Let $\omega \in \bar{\Omega}_{1}, l \in \mathbb{Z}, k \in \mathbb{N}$, then by (3.4)

$$
\mathbb{P}\left\{\omega: N_{1}(l, \omega)>k\right\} \leqslant \sum_{n=k}^{\infty} \mathbb{P}\left(\Omega_{\delta, n, \pm}\right) \leqslant \sum_{n=k}^{\infty} 2 m(\sigma) e^{-\delta_{0}(2 n+1)} \leqslant C e^{-\delta_{0}(2 k+1)}
$$

Thus

$$
\mathbb{P}\left\{\omega: N_{1}(l, \omega)>\ln ^{2}|l|\right\} \leqslant C e^{-\delta_{0}\left(2 \ln ^{2}|l|+2\right)}
$$

By Borel-Cantelli lemma, we can get the result. The same argument works for $N_{3}$.

Then we rebuild the criteria for regularity around a singular point $l$.

Lemma 6.2. For a.e. $\omega$ (we denote this set as $\tilde{\Omega}$ ), for any $l$, there exists $N(l, \omega)$, such that for any $n>N(l, \omega)$ and for all $E \in \sigma$ either $l$ or $l+2 n+1$, and either $l$ or $l-2 n-1$ are $\left(\gamma(E)-8 \epsilon_{0}, n, E, \omega\right)$-regular.

Proof. In section 4, we proved that either 0 or $2 n+1$ is $\left(\gamma(E)-8 \epsilon_{0}, n, E, \omega\right)$ singular for all $n>N(\omega)$, with $N(\omega)=\max \left\{N_{1}(\omega), N_{2}(\omega), N_{3}(\omega)\right\}$. Here we set $N(l, \omega)=\max \left\{N\left(T^{l} \omega\right), N\left(T^{-l} \omega\right)\right\}$, and modify $\tilde{\Omega}$ accordingly.

Now, take $\tilde{\Omega}=\tilde{\Omega}_{2} \cup \tilde{\Omega}_{1,3}$ and fix $\omega \in \tilde{\Omega}$. We omit $\omega$ from notations from now on.

By Lemma 6.1 and Lemma [5.5] there exist $L_{1}, L_{2}, L_{3}$ such that for all $|l|>$ $\max \left\{L_{1}, L_{2}, L_{3}\right\}$

for all $E \in \sigma$.

$$
N_{i}(l) \leqslant \ln ^{2}|l|, \quad \forall i=1,2,3
$$

Let $l_{E}$ be a position of the maximum point of $\varphi_{E}$. Take $L_{4}$ with $\ln ^{2} L_{4} \geqslant$ $\left[\frac{\ln 2}{\gamma(E)-8 \epsilon_{0}}\right]+1$. For any $n \geqslant \ln ^{2} L_{4}$, and any e.v. $E, l_{E}$ is naturally $\left(\mu-8 \epsilon_{0}, n, E\right)-$ singular by (2.2).

Let $L=\max \left\{L_{1}, L_{2}, L_{3}, L_{4}\right\}, N(l):=\max \left\{N_{1}(l), N_{2}(l), N_{3}(l), \frac{\ln 2}{\gamma(E)-8 \epsilon_{0}}\right\}$. Then for any $|l|>L$,

$$
N(l) \leqslant \ln ^{2}|l|
$$

If $\left|l_{E}\right|>L$, then for any $n \geqslant N\left(l_{E}\right), l_{E}$ is $\left(\gamma(E)-8 \epsilon_{0}, n, E\right)$-singular, so $x=$ $l_{E} \pm(2 n+1)$ is $\left(\gamma(E)-8 \epsilon_{0}, n, E\right)$-regular. By (2.2), for any $\left|x-l_{E}\right| \geqslant N\left(l_{E}\right)$

$$
\left|\varphi_{E}(x)\right| \leqslant 2 e^{-\left(\gamma(E)-8 \epsilon_{0}\right)\left|x-l_{E}\right|}
$$

Since $\varphi_{E}$ is normalized, in fact for all $x$,

$$
\left|\varphi_{E}(x)\right| \leqslant 2 e^{\left(\gamma(E)-8 \epsilon_{0}\right) N\left(l_{E}\right)} e^{-\left(\gamma(E)-8 \epsilon_{0}\right)\left|x-l_{E}\right|}
$$

By (6.1), for any $\epsilon$,

$$
\left|\varphi_{E}(x)\right| \leqslant 2 e^{\left(\gamma(E)-8 \epsilon_{0}\right) \ln ^{2}\left(1+\left|l_{E}\right|\right)} e^{-\left(\gamma(E)-8 \epsilon_{0}\right)\left|x-l_{E}\right|}
$$


If $\left|l_{E}\right| \leqslant L$, for any $\epsilon$, for $n \geqslant N\left(l_{E}\right)$, we use the same argument as (6.2) and get

$$
\left|\varphi_{E}(x)\right| \leqslant 2 e^{-\left(\gamma(E)-8 \epsilon_{0}\right)\left|x-l_{E}\right|} \leqslant 2 e^{\epsilon \ln ^{2}\left(1+\left|l_{E}\right|\right)} e^{-\left(\gamma(E)-8 \epsilon_{0}\right)\left|x-l_{E}\right|}
$$

While for $n \leqslant N_{l_{E}}$, set $M_{2 \epsilon}=\min _{k \in[-L, L],|x-k|<N(k)}\left\{e^{\epsilon \ln ^{2}(1+|k|)} e^{-\left(\gamma(E)-8 \epsilon_{0}\right)|x-k|}\right\}$ and $C_{2 \epsilon}=M_{2 \epsilon}^{-1}$. Then for all $\left|x-l_{E}\right|<N\left(l_{E}\right)$,

$$
\left|\varphi_{E}(x)\right| \leqslant 1 \leqslant C_{2 \epsilon} e^{\epsilon \ln ^{2}\left(1+\left|l_{E}\right|\right)} e^{-\left(\gamma(E)-8 \epsilon_{0}\right)\left|x-l_{E}\right|}
$$

Thus for $C_{\epsilon}=\max \left\{2, C_{2 \epsilon}\right\}$, (6.3) (6.4) and (6.5) provide SULE.

\section{ACKNOWLEDGMENTS}

This research was partially supported by the NSF DMS-1401204. X. Z. is grateful to Wencai Liu for inspiring thoughts and comments for Sec. 5. We also thank Barry Simon for his encouragement.

\section{REFERENCES}

[1] René Carmona, Abel Klein, and Fabio Martinelli. Anderson localization for Bernoulli and other singular potentials. Communications in Mathematical Physics, 108(1):41-66, 1987.

[2] Carol Shubin, Ramin Vakilian, and Thomas Wolff. Some harmonic analysis questions suggested by Anderson-Bernoulli models. Geometric and Functional Analysis, 8(5):932-964, 1998.

[3] Jürg Fröhlich and Thomas Spencer. Absence of diffusion in the Anderson tight binding model for large disorder or low energy. Comm. Math. Phys., 88(2):151-184, 1983.

[4] Henrique von Dreifus and Abel Klein. A new proof of localization in the Anderson tight binding model. Communications in Mathematical Physics, 124(2):285-299, 1989.

[5] Svetlana Jitomirskaya. Metal-insulator transition for the almost Mathieu operator. Annals of Mathematics, 150(3):1159-1175, 1999.

[6] Jurg Fröhlich, Thomas Spencer, and Peter Wittwer. Localization for a class of one dimensional quasi-periodic schrödinger operators. Communications in mathematical physics, 132(1):5-25, 1990.

[7] Ya Sinai. Anderson localization for one-dimensional difference Schrödinger operator with quasiperiodic potential. Journal of statistical physics, 46(5):861-909, 1987.

[8] Jean Bourgain and Michael Goldstein. On nonperturbative localization with quasi-periodic potential. Annals of Mathematics, 152(3):835-879, 2000.

[9] Jean Bourgain. Green's Function Estimates for Lattice Schrödinger Operators and Applications.(AM-158). Princeton University Press, 2004.

[10] Valmir Bucaj, David Damanik, Jake Fillman, Vitaly Gerbuz, Tom VandenBoom, Fengpeng Wang, and Zhenghe Zhang. Localization for the one-dimensional Anderson model via positivity and large deviations for the lyapunov exponent. arXiv preprint arXiv:1706.06135, 2017.

[11] Jean Bourgain and Wilhelm Schlag. Anderson localization for Schrödinger operators on $\mathrm{n}$ with strongly mixing potentials. Communications in Mathematical Physics, 215(1):143-175, 2000 .

[12] Anton Gorodetski and Victor Kleptsyn. Parametric Fürstenberg theorem on random products of $\operatorname{sl}(2, r)$ matrices.

[13] Walter Craig, Barry Simon, et al. Subharmonicity of the Lyaponov index. Duke Mathematical Journal, 50(2):551-560, 1983.

[14] Hans L Cycon, Richard G Froese, Werner Kirsch, and Barry Simon. Schrödinger operators: with application to quantum mechanics and global geometry. Springer, 2009.

[15] Émile Le Page. Théoremes limites pour les produits de matrices aléatoires. In Probability measures on groups, pages 258-303. Springer, 1982.

[16] Tsay, Jhishen. Some uniform estimates in products of random matrices. Taiwanese Journal of Mathematics, pages 291-302, 1999.

[17] Artur Avila and Svetlana Jitomirskaya. The ten martini problem. Annals of Mathematics, pages 303-342, 2009. 
[18] Pedro Duarte, Silvius Klein, et al. Lyapunov exponents of linear cocycles. Atlantis Series in Dynamical Systems (Springer, 2016), 2016.

[19] Rafael del Rio, Svetlana Jitomirskaya, Yoram Last, and Barry Simon. Operators with singular continuous spectrum, IV. Hausdorff dimensions, rank one perturbations, and localization. Journal d'Analyse Mathématique, 69(1):153-200, 1996.

Department of Mathematics, University of California, Irvine 